\title{
Theoretical background and the flow fields in downhole liquid-liquid hydrocyclone (LLHC)
}

\author{
Harrison Osei ${ }^{1, \text { a }}$, Hussain H. Al-Kayiem ${ }^{2}$ and Fakhruldin Bin Mohd Hashim ${ }^{3}$ \\ 1, 2, 3 Department of Mechanical Engineering, UTP, 31750 Tronoh, Perak, Malaysia
}

\begin{abstract}
Hydrocyclone system for downhole oil-water separation provides an effective technique of enhancing the economic viability of higher water-cut wells while at the same time reducing the risk of environmental pollution. This paper describes the hydrodynamics of the liquid-liquid hydrocyclones and the flow fields within it are paramount for achieving successful separation process. Some of the important hydrodynamic flow phenomenon within the liquid-liquid hydrocyclone and how they influence the separation efficiency of water/oil was analyzed through analytical solution. The properties of the liquids were based on Bayan offshore field measured properties. The results indicated that there are two swirling zones separated by stagnant flow field. The inner is the light liquid zone, while the outer is the heavy liquid zone.
\end{abstract}

\section{Introduction}

The hydrocyclone nowadays finds many applications in the separation of solid-liquid streams, liquidliquid streams and gas-liquid streams [1]. Those that are used to separate suspended liquid droplets from another liquid stream are termed LLHC. LLHC can be employed downhole for the separation of oil-water products, due to its compactness, absence of moving parts and no chemical additives. This technology allows the produced water to be separated downhole and re-injected into the same formation at suitable depths. It again reduces the volume of produced water to the surface, water treatment cost and possibility of pollution at the surface. The fluids within the cyclone undergo some flow patterns before achieving separation. This paper presents the mathematical models that can be used to predict flow fields within LLHC and their influence on the separation performance of the system.

\section{Operational Principles of LLHC}

A schematic drawing of a LLHC is shown in Fig. 1. It is made up of a combination of conical and cylindrical sections or parts. The upper cylindrical part is closed at the top by a cover, through which is the vortex finder. According to Bower et al. [1], the fluid produced when fed tangentially into the upper cylindrical portion of the LLHC creates a swirling movement of the fluid making the heavier fluid fraction, water, to spin to the outside of the LLHC whereas the lighter fluid fraction, oil, to migrate toward the core of the LLHC. Gomez et al. [2] pointed out that, this swirl movement causes the flow pattern within the hydrocyclone to consist of a spiral within another spiral. The inner spiral moves upward with the outer spiral moving downward. [2] showed how the forced vortex, existing in the area close to the axis of the LLHC, and the free-like vortex, existing at the outer area, behave and influence separation within the LLHC. The outer vortex moves downwards to the underflow opening, with the inner vortex flowing in a reverse direction to the overflow outlet. The reverse flow in the LLHC is due to the high swirling intensity at the inlet which increases the centrifugal force and causes the pressure to be high near the wall region and very low toward the core region. Subjecting the underflow outlet to a higher pressure than that of the overflow, will cause the concentrated oil core of

This is an Open Access article distributed under the terms of the Creative Commons Attribution License 2.0, which permits unrestricted use, distribution, and reproduction in any medium, provided the original work is properly cited. 
the vortex to be forced to flow counter currently to the main flow $[1,2]$. The centrifugal force developed accelerates the settling rate of the fluid particles thereby separating them according to size, shape, and difference in density. Due to the action of the drag force, the oil moves to the core region of the LLHC and is caught by the reverse flow to be separated through the vortex finder. The water moves to the walls of the LLHC and migrates to the apex opening [2, 3].

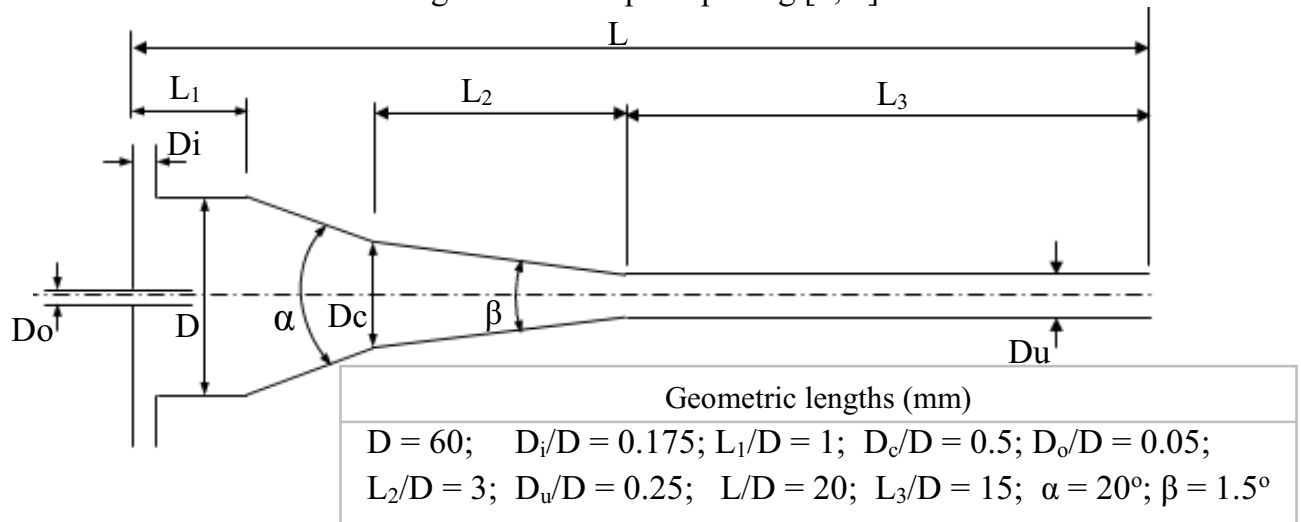

Figure 1: Schematic diagram of LLHC (modified after Colman \& Thew)[2]

In this work, the mathematical models that describe the hydrodynamics of the LLHC and the flow patterns are presented. They were coded in excel spreadsheet taking into account the geometry of Fig. 1 and fluid properties similar to Bayan oil fields - Malaysia, to obtain information about the hydrodynamics and the flow fields likely to be observed in the course of using the LLHC.

\section{Swirl intensity}

The swirl intensity, $\Omega$, is produced by the tangential inlet of the LLHC and is defined as the ratio of the rate of tangential momentum flux to the total momentum flux at a specific axial location[4]. However, researchers have made some modifications to the swirl intensity correlation to help better predict it. Chang and Dhir correlation [5] shown in Eq. 1 took into account only the momentum flux at both the inlet slot and the characteristic diameter position. To incorporate the fluid properties and the inlet configuration, Mantilla [5] modified Chang and Dhir's model and ended up with Eq. 2. Caldentey correlation [6] shown in Eq. 3 included the semi angle, $\beta$, of the LLHC. By combining Caldentey and Erdal correlations, Gomez [6] ended up with Eq. 4 as the correlation for swirl intensity.

$$
\begin{gathered}
\Omega=1.48\left(\frac{M_{t}}{M_{T}}\right)^{0.93} \operatorname{EXP}\left[-0.113\left(\frac{M_{t}}{M_{T}}\right)^{0.35}\left(\frac{z}{D c}\right)^{0.7}\right] \text { for } \frac{z}{D c} \geq 2 \\
\Omega=1.48\left(\frac{M_{t}}{M_{T}} I^{2}\right)^{0.93} \operatorname{EXP}\left[-\left(\frac{1}{2}\right)\left(\frac{M_{t}}{M_{T}} I^{4}\right)^{0.35}\left(\frac{1}{\operatorname{Re}}\right)^{0.16}\left(\frac{z}{D c}\right)^{0.7}\right] \text { for } \frac{z}{D c} \geq 2 \\
\Omega=1.48\left(\frac{M_{t}}{M_{T}} I^{2}\right)^{0.93}\left(1+1.2 \tan (\beta)^{0.15}\right) * \\
\operatorname{EXP}\left[-\left(\frac{1}{2}\right)\left(\frac{M_{t}}{M_{T}} I^{4}\right)^{0.35}\left(\frac{1}{\operatorname{Re}_{z}}\right)^{0.16}\left(\frac{z}{D c}\right)^{0.7}\left(1+1.2 \tan (\beta)^{0.12}\right)\right]
\end{gathered}
$$




$$
\begin{gathered}
\Omega=0.49 \operatorname{Re}^{0.118}\left(\frac{M_{t}}{M_{T}} I^{2}\right)^{0.93}\left(1+1.2 \tan (\beta)^{0.15}\right) * \\
E X P\left[-\left(\frac{1}{2}\right)\left(\frac{M_{t}}{M_{T}} I^{4}\right)^{0.35}\left(\frac{1}{\mathrm{Re}_{z}}\right)^{0.16}\left(\frac{z}{D c}\right)^{0.7}\left(1+2 \tan (\beta)^{0.12}\right)\right]
\end{gathered}
$$

where

$$
\frac{M_{t}}{M_{T}}=\frac{A_{c}}{A_{i s}} \quad \text { (5) } \quad \text { and } \quad \operatorname{Re}_{z}=\frac{\rho_{c} U_{a v z} D_{z}}{\mu_{c}}
$$

For Eq. 2 and Eq. 4, $I=1-\exp \left(-\frac{n}{2}\right)$ and for Eq. 3, $I=1-\exp (-n)$.

$\rho_{c}$ and $\mu_{c}$ are the density and viscosity of the continuous phase respectively; $\mathrm{z}$ is the axial position; $A c$ and $\mathrm{A}_{\mathrm{is}}$ are the cross-sectional area at the characteristic diameter Dc and at the inlet slot respectively; $U_{a v z}$ is the average axial velocity at z. For Eq. 3 and Eq. $4, \mathrm{n}=1.5$ for twin inlets and for Eq. 2, $\mathrm{n}=2$ for twin inlets.

\section{Velocity Field within LLHC}

The velocity field in the hydrocyclone has major impact on the separation of fluid. The swirl intensity is known to relate the local axial velocity to the tangential velocity. This means that if the swirl intensity can be predicted for a particular axial position, the prediction of the velocity profiles within the cyclone becomes easier [3, 4].

\subsection{Tangential Velocity}

The tangential velocity has been known and confirmed experimentally to be a combination of a forced vortex and free-like vortex. The former exists near the axis of the hydrocyclone with the latter existing near the walls of the hydrocyclone. Due to the tangential entry of the fluid into the LLHC system, the fluid swirls and produces centrifugal forces which help separate the fluid particles based on density difference with the heaviest to the lightest particle arranged from the cyclone's wall to its center respectively. They proposed the following equation for the tangential velocity profile $[3,4]$ :

$$
\frac{w}{U_{a v c}}=\frac{T_{m}}{\left(r / R_{c}\right)}\left\{1-\exp \left[-B\left(\frac{r}{R_{c}}\right)^{2}\right]\right\}
$$

where, $U_{a v c}$ and $R_{c}$ are the is the average axial velocity and the radius at the characteristic diameter, Dc, respectively; and $\mathrm{r}$ is the radial position. $\mathrm{T}_{\mathrm{m}}=\Omega ; \mathrm{B}=245.8 \Omega^{-2.35}$ (Twin inlets)

\subsection{Axial Velocity}

The axial velocity profile can be predicted by the use of a third-order polynomial equation after having imposed proper boundary conditions. The general form is as follows [3]:

$$
u(r)=a_{1} r^{3}+a_{2} r^{2}+a_{3} r+a_{4} .
$$

where $\mathrm{a}_{1}, \mathrm{a}_{2}, \mathrm{a}_{3}$ and $\mathrm{a}_{4}$ are constants. The boundary conditions are as follow:

1. $\frac{d u\left(r=R_{z}\right)}{d r}=0$

2. $u\left(r=r_{\text {rev }}\right)=0$

3. $\frac{d u(r=0)}{d r}=0$

4. $\quad 2 \pi \rho_{c} \int_{0}^{R_{z}} u(r) r d r=U_{a v z} \rho_{c} \pi R_{z}^{2}$
(The wall velocity considered maximum);

(Velocity at the location of reverse flow, $\mathrm{r}_{\text {rev, }}$ considered zero);

(The velocity been symmetrical about the LLHC axis);

(Conservation of mass). 
If the boundary conditions are substituted into Eq. 8, will yield the axial velocity profile relation [3]:

$$
\begin{aligned}
& \frac{u(r)}{U_{a v z}}=\frac{2}{C}\left(\frac{r}{R_{z}}\right)^{3}-\frac{3}{C}\left(\frac{r}{R_{z}}\right)^{2}+\frac{0.7}{C}+1 \\
& C=\left(\frac{r_{r e v}}{R_{z}}\right)^{2}\left(3-2\left(\frac{r_{r e v}}{R_{z}}\right)\right)-0.7 \\
& \frac{r_{r e v}}{R_{z}}=0.21 \Omega^{0.3}
\end{aligned}
$$

\subsection{Radial Velocity}

The radial velocity, $\mathrm{v}$, of the continuous phase is very small, and as a result of that many studies choose to neglect it. It can however be calculated by using Eq. 12[3, 4].

$$
v=-\frac{r}{R_{z}} u \tan (\beta)
$$

\section{Results and Discussion}

The comparison between the various mathematical models for swirl intensity and how they fit some selected DOWS data from literature is shown in Fig. 2. From the figure, Chang and Dhir's model over predicts the swirl intensity at $\mathrm{z} / \mathrm{Dc}<10$ but predict the data well at $\mathrm{z} / \mathrm{Dc}>20$. Gomez and Mantilla models fairly fit the data with Caldentey's model not fitting.

The velocity profiles predicted by the models were compared with some field test data from DOWS operations. Fig. 3 shows the tangential velocity comparison of the various models with field experimental data. The $\mathrm{y}$-axis represents the cyclone axis whereas the $\mathrm{x}$-axis represents the radial position. All the models show the Rankine Vortex shape, the combination of forced vortex near the LLHC axis and the free vortex near the wall. From the figure, Gomez model seems to give a good fit than the others.

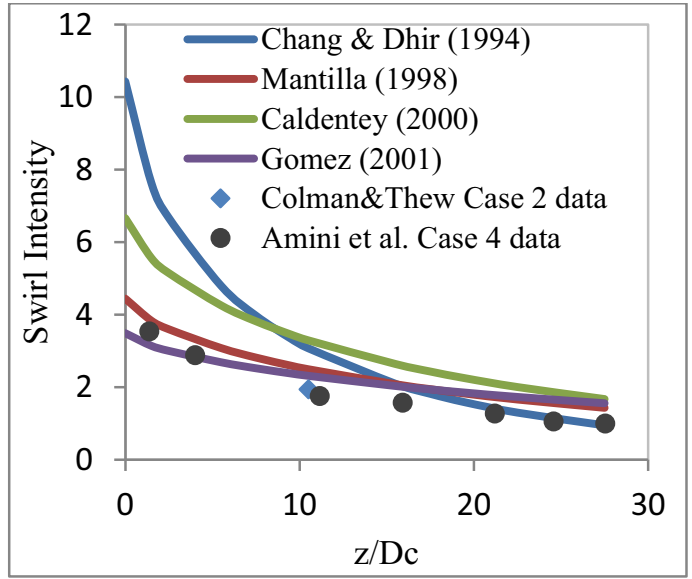

Figure 2: Swirl intensity comparison

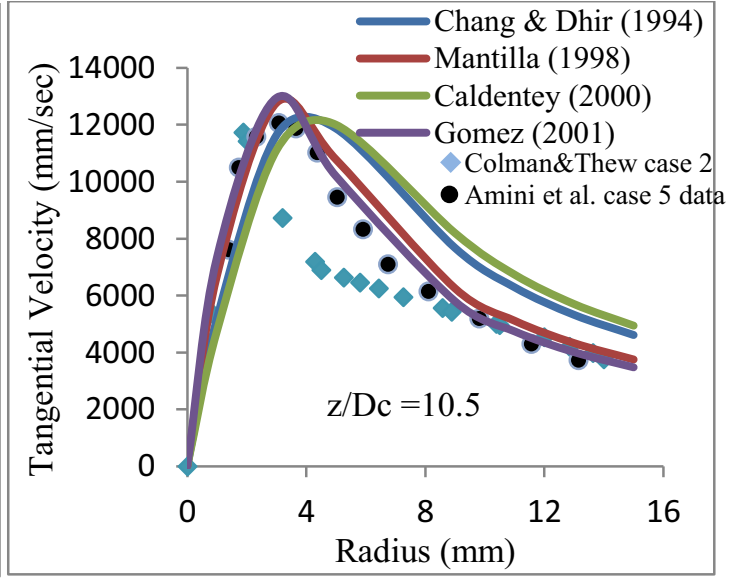

Figure 3: Tangential velocity comparison

The axial velocity profile for the flow within the LLHC is presented in Fig. 4. The positive values of the axial velocities correspond to downward flow while the negative values correspond to the reverse flow. The model matches well the data points for the downward flow and not good in the reverse flow region. However, in computing the separation efficiency of the LLHC, the reverse flow velocity is not so important but the radial position at which there is zero velocity. This reason is well explained in Fig. 5.

Fig. 5 presents the profile for the radial positions within the LLHC at various axial locations where the axial velocity is zero. The y-axis represents the axis of the LLHC and the x-axis represents the 
radial position. By connecting all the radial positions of zero velocity at the different axial positions within the LLHC give the profile (plane) shown in Fig. 5. Fluids existing between the two profile lines constitute the upward spiral and will be collected as overflow. Those fluids existing outside the profile lines will move downwards and be collected as underflow. Those existing on the profile lines have fifty-fifty chance of going to either outlet.

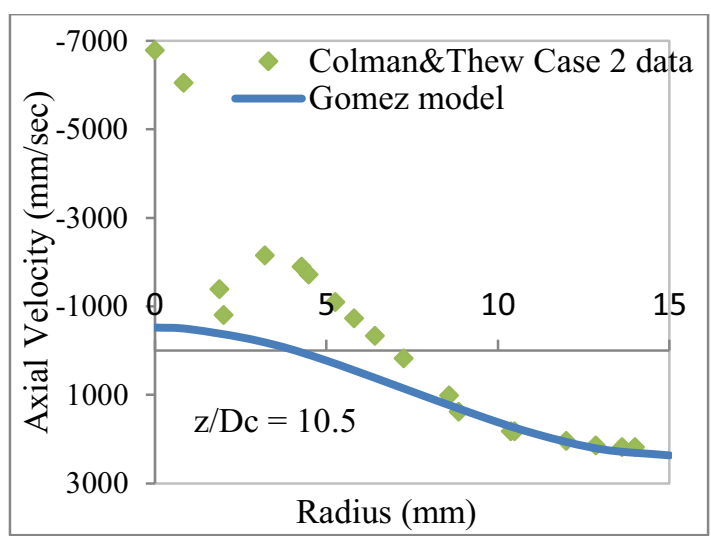

Figure 4: Axial velocity comparison



Figure 5: Profile for the radial positions of zero axial velocity

\section{Conclusion}

This work has shown that an in-depth knowledge of the hydrodynamics and the flow patterns within the LLHC are very important and should not be underestimated. The reverse radius is an important factor in the separation efficiency of the LLHC. Gomez model agrees with most of the experimental data obtained from literature. An experiment must therefore be performed using the proposed LLHC design modified after Colman and Thew and the data that will be obtained used to validate the mathematical correlations proposed by the aforementioned scholars.

\section{Acknowledgement}

The authors acknowledge Universiti Teknolgi PETRONAS for the financial and technical support to produce this paper under the research grant YUTP - FRG 015-3AA-A73.

\section{References}

[1] B. E. Bowers, R. F. Brownlee, and P. J. Schrenkel, "Development of a downhole oil/water separation and reinjection system for offshore application”. SPE Prod. \& Facilities, vol.15, no. 2, pp. 115-122, (2000).

[2] C. Gomez, J. Caldentey, and S. Wang, L. Gomez, R. Mohan, and O. Shoham, "Oil/Water Separation in Liquid/Liquid Hydrocyclones (LLHC): Part 1 - Experimental Investigation”, SPE J., pp. 353-361, (2002).

[3] J. Caldentey, C. Gomez, S. Wang, and L. Gomez, R. Mohan, and O. Shoham, "Oil/Water Separation in Liquid/Liquid Hydrocyclones (LLHC): Part 2 - Mechanistic Modeling”, SPE J., pp. 362-372, (2002).

[4] D. M. Amini, M. Golkar and F. Esmaeilzadeh, "Mathematical modelling of a hydrocyclone for the down-hole oil-water separation (DOWS)" Chem. Eng. Research and Design, vol. 90., pp. 21862195, (2012).

[5] I. S. Mantilla, "Bubble Trajectory Analysis in Gas-Liquid Cylindrical Cyclone Separators", MSc. Dissertation, Dept. Petroleum Eng., University of Tulsa, (1998).

[6] C. H. Gomez, "Oil-Water Separation in Liquid-Liquid Hydrocyclones (LLHC) Experiment and Modeling”, MSc. Dissertation, Dept. Petroleum Eng., University of Tulsa, (2001). 